\title{
A simple methodology to detect and quantify wind power ramps
}

\author{
Bedassa R. Cheneka ${ }^{1}$, Simon J. Watson ${ }^{1}$, and Sukanta Basu ${ }^{2}$ \\ ${ }^{1}$ Wind Energy Section, Faculty of Aerospace Engineering, \\ Delft University of Technology, Delft, the Netherlands \\ ${ }^{2}$ Faculty of Civil Engineering and Geosciences, Delft University of Technology, Delft, the Netherlands \\ Correspondence: Bedassa R. Cheneka (b.r.cheneka@ @udelft.nl)
}

Received: 12 March 2020 - Discussion started: 6 April 2020

Revised: 18 August 2020 - Accepted: 5 November 2020 - Published: 16 December 2020

\begin{abstract}
Knowledge about the expected duration and intensity of wind power ramps is important when planning the integration of wind power production into an electricity network. The detection and classification of wind power ramps is not straightforward due to the large range of events that is observed and the stochastic nature of the wind. The development of an algorithm that can detect and classify wind power ramps is thus of some benefit to the wind energy community. In this study, we describe a relatively simple methodology using a wavelet transform to discriminate ramp events. We illustrate the utility of the methodology by studying distributions of ramp rates and their duration using 2 years of data from the Belgian offshore cluster. This brief study showed that there was a strong correlation between ramp rate and ramp duration, that a majority of ramp events were less than $15 \mathrm{~h}$ with a median duration of around $8 \mathrm{~h}$, and that ramps with a duration of more than a day were rare. Also, we show how the methodology can be applied to a time series where installed capacity changes over time using Swedish onshore wind farm data. Finally, the performance of the methodology is compared with another ramp detection method and their sensitivities to parameter choice are contrasted.
\end{abstract}

\section{Introduction}

Rapid changes in wind speed can cause ramps in the wind power production of a wind farm. With plans to install a large amount of capacity in the North Sea, understanding swings in offshore wind farm power production will become important for wind farm and network operators to manage the integration of wind power into the electricity system. In their development of a wind power variability index, Kiviluoma et al. (2014); Kiviluoma et al. (2016) distinguish between three different timescales which are of importance to system operators. The first of these is sub-hourly up to $2 \mathrm{~h}$ where load following and frequency control is required. The second and that where wind power generation is stated to cause most ramping is for timescales of between $1 \mathrm{~h}$ and around $15 \mathrm{~h}$. For these timescales, there can be significant impact on thermal plant start-ups and shutdowns, though this depends on the characteristics of the plant installed in the particular power system. The final ramping timescales of relevance are between $10 \mathrm{~h}$ and around a day. This is of relevance where large-scale storage is required such as hydro pump storage. An understanding of the prevalence and magnitude of ramps across a range of timescales is thus important.

Wind power ramps are influenced by the dynamics of atmosphere-ocean systems which could be either mesoscale or synoptic-scale. Therefore, meteorological systems that evolve over time play a significant role in the occurrence of power ramps (Marquis et al., 2011). Low-pressure systems, cold fronts, low-level jets, thunderstorm outflows, and dry lines can cause ramp-up (increasing wind) events (Sevlian and Rajagopal, 2013; DeMarco and Basu, 2018), whereas ramp-down (decreasing wind) events occur due to the reduction or reversal of these physical processes (Ferreira et al., 2011). Short-duration (rapid) power ramps are mainly influenced by mesoscale systems, whereas synoptic systems tend to be responsible for longer-duration power ramps (Drew et al., 2018). 
There is no accepted definition or classification of wind power ramps except that they are manifested in terms of a significant change in production over a relatively short time. The quantification of the duration and magnitude of wind power ramps has been explored by several scholars. Most of the studies set thresholds with respect to the rated power of the wind farm to detect wind power ramps. One such definition (Bossavy et al., 2010; Zhang et al., 2017) defines a ramp as a minimum change in wind farm output $\Delta P$ as a fraction of the rated wind power $P_{\text {rated }}$ of the wind farm over a period of time $(\Delta t)$. Different researchers consider different rates of change to define ramps; for example, Cutler et al. (2007) define a power ramp when there is a change in wind power production of $75 \%$ of $P_{\text {rated }}$ within a $\Delta t$ of $3 \mathrm{~h}$ or $65 \%$ of $P_{\text {rated }}$ within a $\Delta t$ of $1 \mathrm{~h}$. In contrast, Bossavy et al. (2010) define a wind power ramp when there is a change in wind power of $50 \%$ of $P_{\text {rated }}$ over $1 \mathrm{~h}$. Other researchers such as Bianco et al. (2016) and Gallego-Castillo et al. (2015) use still different percentage changes in wind power and time ranges to define wind power ramps. There have been studies to detect power ramps without using any pre-defined change in wind power relative to rated power and time. An optimised swinging-door algorithm was used by Zhang et al. (2017) to extract ramps where the ramp definition parameters related to power change and timescale could be easily adapted. An optimal method based on scoring functions (Sevlian and Rajagopal, 2013) was used to detect ramps of varying lengths at a US wind farm. These authors used a piecewise linear trending fit to remove short-term stochastic fluctuations.

Even though there has been a significant body of work to detect wind power ramps, it is clear that there is no precise consensus as to the definition of a ramp. Indeed, it may be necessary to extract information about a range of power ramp events depending on the requirements of the wind farm operator or the utility as described above. What is required is a robust method which can extract ramps of arbitrary magnitude and duration and to discriminate above the incoherent stochastic noise level. In this paper, we propose an improved method to discriminate ramp events above incoherent stochastic variations using wavelets. Wavelets have been used in the past to extract ramp events from time series, e.g. Hannesdóttir and Kelly (2019); Ji et al. (2015); Gallego et al. (2014); Coughlin et al. (2014). We build on this work by demonstrating how a wavelet transform can be used in conjunction with the generation of wind power surrogates to give a robust method for the detection of wind power ramps of varying magnitude and duration. Rather than relying on fixed power or timescale thresholds, the methodology uses a method of discrimination based on statistical thresholds. We illustrate the methodology and its application using data from Belgian offshore and Swedish onshore wind farms. Firstly, we describe the methodology and illustrate its application using a $10 \mathrm{~d}$ period of data. Next, the sensitivity of the discrimination of ramps from natural stochastic variation is investigated using a longer period to generate the surrogate distribu- tions. Then, we show the utility of the approach in terms of characterising the distribution of ramp rates, their duration, and their diurnal-seasonal variation using 2 years of the offshore wind power data. Next, the versatility of the methodology is demonstrated for a non-stationary time series where installed capacity changes over time. Finally, we compare the methodology with another commonly used approach, namely the min-max method (Bianco et al., 2016).

\section{The wind farm data}

The Belgian transmission system operator, Elia, makes available $15 \mathrm{~min}$ power output data for the aggregated fleet of Belgian onshore and offshore turbines (Elia, 2020). In this work, we have used offshore data over a period of 2 years from 2015 to 2016 when the combined Belgian offshore wind power capacity was $712 \mathrm{MW}$. For simplicity, the $15 \mathrm{~min}$ values were normalised to the total capacity before analysis to create a time series of values $P(t)$. In addition, we make use of Swedish onshore hourly wind power data for the period 2000-2001 aggregated within the SE1 price region (SCB, 2017). The installed capacity in this region increased from about 500 to $1300 \mathrm{MW}$ over this period. Further details of this dataset can be found in EEM20 (2020).

\section{Wavelet decomposition}

The continuous wavelet transform (CWT) can be used to decompose a series of data using a mother wavelet function $(\psi)$ by varying its dilation and translation. A mother wavelet function with scale $a$ and position $b$ can be defined as (Mallat, 2009)

$\psi^{a, b}(t)=\psi\left(\frac{t-b}{a}\right)$.

The CWT $W(a, b)$ of a signal $X(t)$ is produced by the convolution of the mother wavelet function over a range of scales and positions:

$W(a, b)=\frac{1}{\sqrt{a}} \int_{-\infty}^{\infty} X(t) \psi\left(\frac{t-b}{a}\right) \mathrm{d} t$.

A wavelet transform is thus able to localise the scales of a series of data in time which makes it a useful function to detect and characterise wind power ramps. We use the Daubechies level-1 (Haar) mother wavelet to decompose the time series of power values. This wavelet is useful to detect abrupt changes in a level which might be expected to occur during a ramp event.

Using values over a $10 \mathrm{~d}$ period, 27 January to 7 February 2015, taken from the Belgian offshore wind power data, a CWT was applied, and the results are shown in Fig. 1 comparing the original time series (a) with the corresponding 
(a)

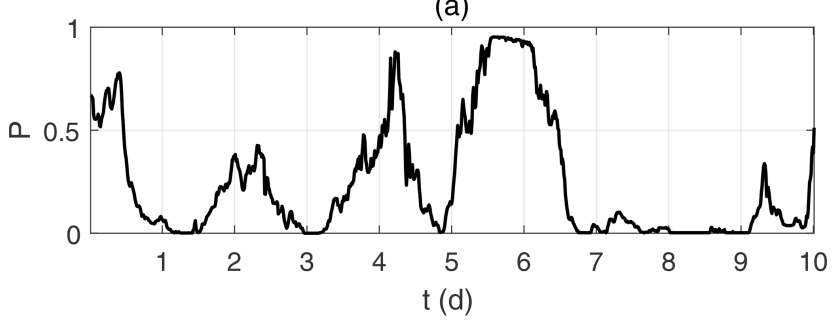

(b)

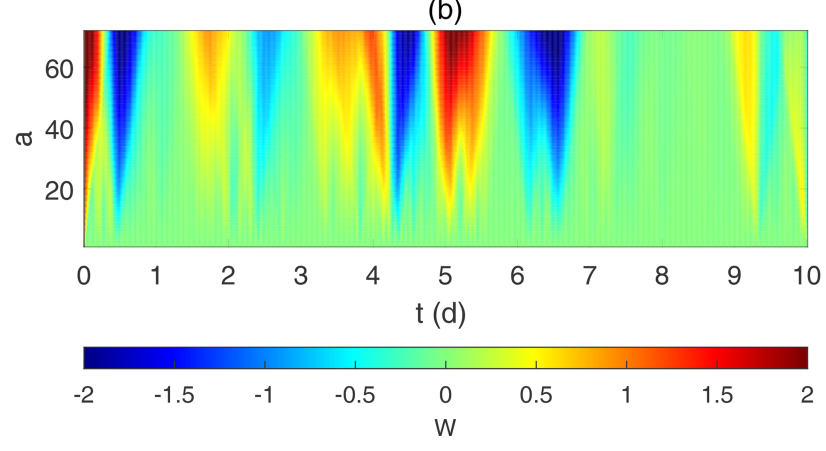

Figure 1. (a) Aggregated Belgian offshore wind power output over the period 27 January to 7 February 2015. (b) Continuous wavelet transform of the wind power data using the Haar mother wavelet.

CWT values (b). It can be seen that a high magnitude of $W$ corresponds to a strong power ramp. A similar finding has been reported elsewhere (Gallego et al., 2014; Hannesdóttir and Kelly, 2019). However, what is not clear is what magnitude of $W$ can be considered a ramp above the incoherent stochastic variations in wind power. In the following section, we consider how to discriminate ramps above such stochastic variations.

\section{Discrimination of ramp events}

Random shuffling is a technique to generate surrogate data from an original time series which preserves limited statistical properties of the original data, namely their distribution. However, it destroys the auto-correlation within a time series. Randomly shuffled surrogates have been used to test for non-linearity in a time series (Theiler et al., 1992). They have also been used to test for stationarity in temporal data (Laurent and Doncarli, 1998; Davy and Godsill, 2002; Borgnat and Flandrin, 2009; Guarin et al., 2010; Borgnat et al., 2010). Furthermore, surrogates have been applied to discriminate gusts and other coherent structures from incoherent noise in high-frequency wind speed data (Dunyak et al., 1998; Gilliam et al., 2000).

In Fig. 2, as an example, we analyse a surrogate based on the $10 \mathrm{~d}$ time series shown in Fig. 1a. Figure 2a shows a comparison between the auto-correlation of the original time series of normalised power values, $P(t)$, and that of a randomly shuffled time series of these values, $P^{*}(t)$. It can be seen that any coherent structure in the original data is destroyed. Figure $2 \mathrm{~b}$ shows the continuous wavelet transform of the surrogate time series, $W^{*}(t)$. It can be seen that the lower-frequency (higher-scale value) structure that was seen in Fig. 1b has disappeared and the power in the transformed wavelet spectrum is much more distributed over all scales.

In order to test the hypothesis that the value of a wavelet coefficient represents a ramp event, we generate 100 such randomly shuffled surrogates of normalised wind power, $P_{i}^{*}(t)$, where $i=1$ to 100 , based on the $10 \mathrm{~d}$ of data. For each surrogate time series, the CWT is generated to give a series of coefficients $W_{i}^{*}(a, b)$. These are used to generate distributions of coefficient values (containing $100 \times b$ values) for each scale $a$, against which the CWT coefficient of the original, $W(a, b)$, can be compared. In Fig. 3a-d we show the distributions for $W$ and $W^{*}$ at the scale $a=40$, where we discriminate the $W$ values based on the largest $10 \%, 5 \%$, $2 \%$, and $1 \%$ of $\left|W^{*}\right|$ values, respectively. The threshold values, $\pm W_{\mathrm{T}}^{*}$, are shown for each plot in Fig. 3 .

We then extend this method of discrimination to all the scales of $W$. So, for each scale $a$, we compute a scaledependent threshold $W_{\mathrm{T}}^{*}(a)$ for a specific discrimination level by utilising the $\left|W_{i}^{*}(a, b)\right|$ values from all the surrogates. If the value of $|W(a, b)|$ is greater than this threshold $W_{\mathrm{T}}^{*}(a)$, then the null hypothesis (no ramp) is rejected at the specific discrimination level and the event is assumed to be a wind power ramp. We repeat this for the four different discrimination levels used above, namely the $10 \%, 5 \%, 2 \%$, and $1 \%$ levels.

Figure 4 shows the result of using this approach to discriminate the wind power ramps at each scale. The plot is similar to the bottom plot in Fig. 1, but now values which do not satisfy the criterion to be considered ramps have been removed and are shown as white with different null hypothesis testing. Only the colour-shaded values that satisfy the requirement to be considered wind power ramps for different discrimination levels are shown.

It is then possible to sum $W(a, b)$ over all discriminated scales up to the maximum resolved, $a_{\max }$, at each time step, $t=b$, to calculate mean normalised power ramps, $R(t)$ :

$R(t=b)=\frac{1}{a_{\max }} \sum_{a=1}^{a_{\max }} W_{\mathrm{R}}(a, b)$,

where

$\begin{array}{lll}W_{\mathrm{R}}(a, b)=W(a, b) & \text { when } & |W(a, b)| \geq W_{\mathrm{T}}^{*}(a), \\ W_{\mathrm{R}}(a, b)=0 & \text { when } & |W(a, b)|<W_{\mathrm{T}}^{*}(a) .\end{array}$

Figure 5 shows the original $10 \mathrm{~d}$ time series of wind power values with the normalised ramp values, $R(t)$ superimposed. Power ramps are now clearly defined in terms of both timing and magnitude. Although there is not a large difference in those events which are classified as ramps, the events on days 9 and 10 in particular are excluded at the two highest 
(a)

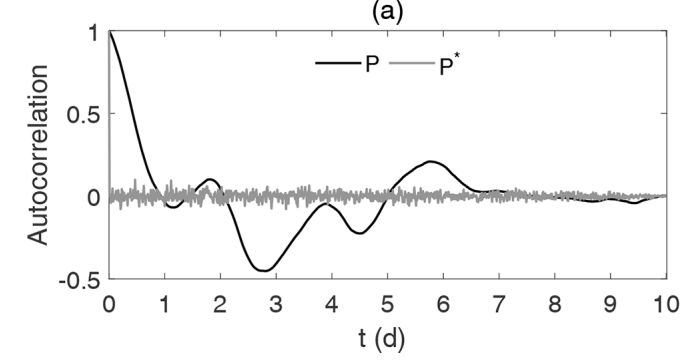

(b)

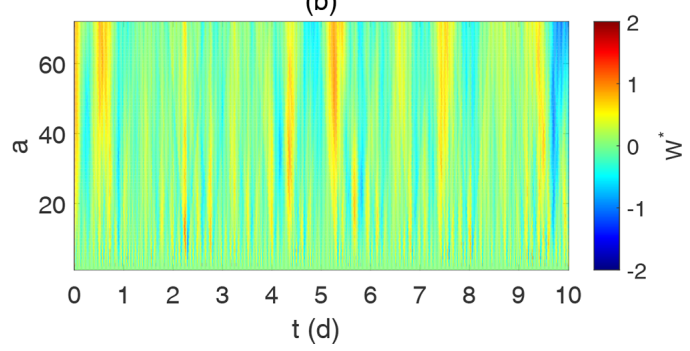

Figure 2. (a) The auto-correlations of the original wind power $(P)$ and the normalised wind power surrogate $\left(P^{*}\right)$; $(\mathbf{b})$ the $\mathrm{CWT}$ of the normalised wind power surrogate.
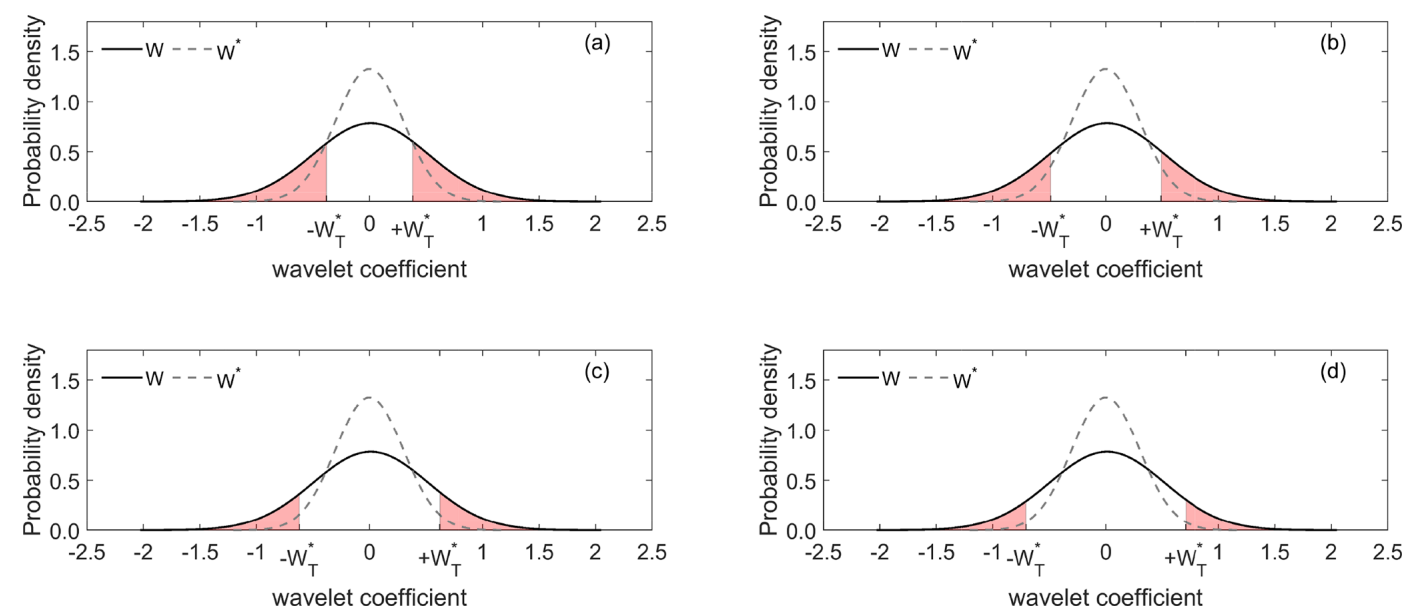

Figure 3. The distributions of $W$ and $W^{*}$ shown at the scale $a=40$. The shaded regions show the $W$ values which are classified as ramp events based on thresholds $\pm W_{\mathrm{T}}^{*}$ set using the highest (a) $10 \%$, (b) $5 \%$, (c) $2 \%$, and (d) $1 \%$ of the $W^{*}$ distribution.

discrimination levels. For the remainder of the paper we have chosen the $10 \%$ level to provide a good balance between the removal of stochastic variation whilst preserving ramp events that would be of relevance from a power system perspective.

\section{Sensitivity to length of surrogate series}

To test the generality of the technique, we consider further testing periods and increase the length of time for which the surrogate distributions are calculated. Three additional $10 \mathrm{~d}$ periods are selected, and for each period, we examine the sensitivity of the results to the length of surrogate, namely, the same $10 \mathrm{~d}$ period and 1 calendar year of values encompassing the $10 \mathrm{~d}$ period. These cases are summarised in Table 1.

As before, for each case, we generate 100 surrogates and the wavelet coefficients $W(a, b)$ are discriminated from the distributions generated using the two different surrogate periods in Table 1. The results are presented in Fig. 6. It can be seen that once again, ramp periods are well discriminated from periods of incoherent stochastic variation. In addition, the results show no difference when using a longer period to generate the surrogates except at the very beginning and end of the time series. This is due to boundary effects inherent in using a convolution function which is integrated over all time and should thus be disregarded in any comparison. The fact that the results show no differences when using an extended surrogate period confirms that the process is filtering out short-term incoherent fluctuations and that a $10 \mathrm{~d}$ period is sufficient to capture these.

\section{Ramp rates and duration}

In this section, we show how the methodology can quantify both ramp rates and their duration, using 2 years of the Belgian offshore wind power data for 2015-2016. We also use this 2-year period to produce the surrogate distributions for deriving the $10 \%$ discrimination level. Firstly, we generate a time series of normalised ramp rates. As can be seen in Figs. 5 and 6, there are discrete periods of ramp-up and rampdown events. For each ramp-up period $k$ and ramp-down pe$\operatorname{riod} l$, we calculate the average ramp-up rate, $R_{\mathrm{u}}^{\prime}(k)$, and average ramp-down rate, $R_{\mathrm{d}}^{\prime}(l)$ : 

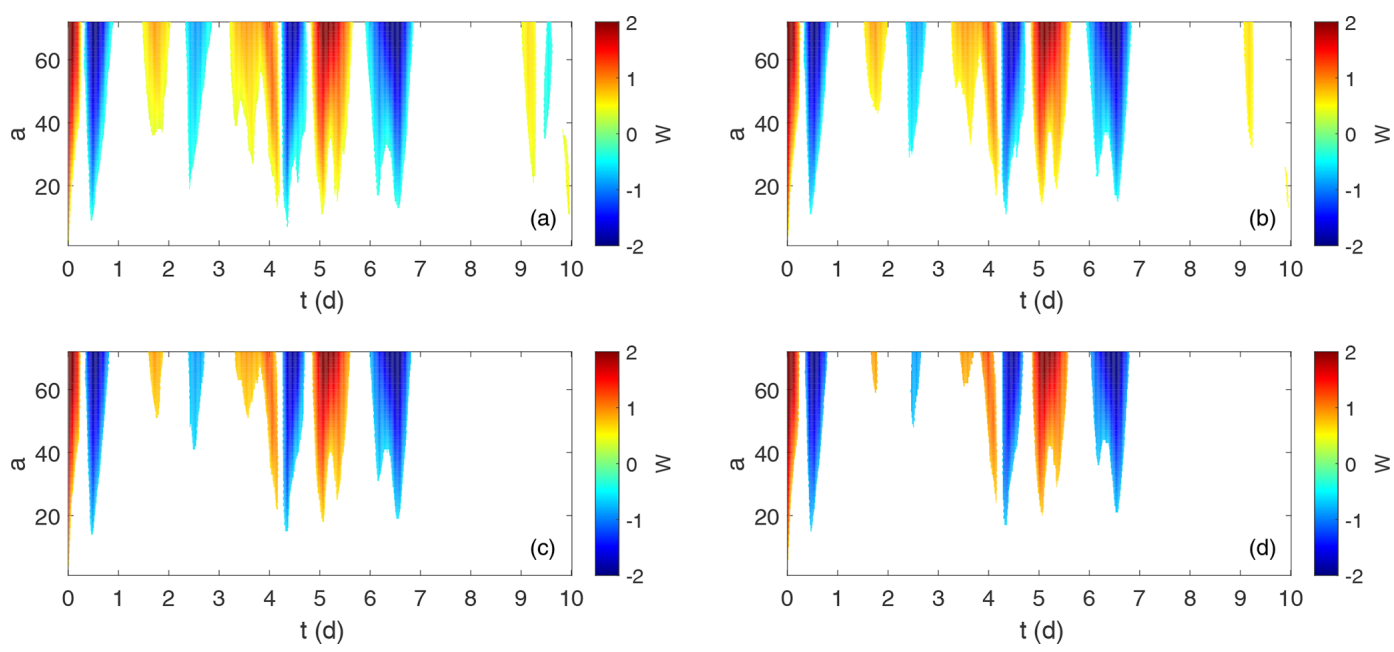

Figure 4. The CWT coefficients $W(a, b)$ of the normalised wind power discriminated from the distribution of $W_{i}^{*}(a, b)$ using the (a) $10 \%$, (b) $5 \%$, (c) $2 \%$, and (d) $1 \%$ discrimination levels. The colour scale is blue (ramp-down events), red (ramp-up events), and white (no ramp).
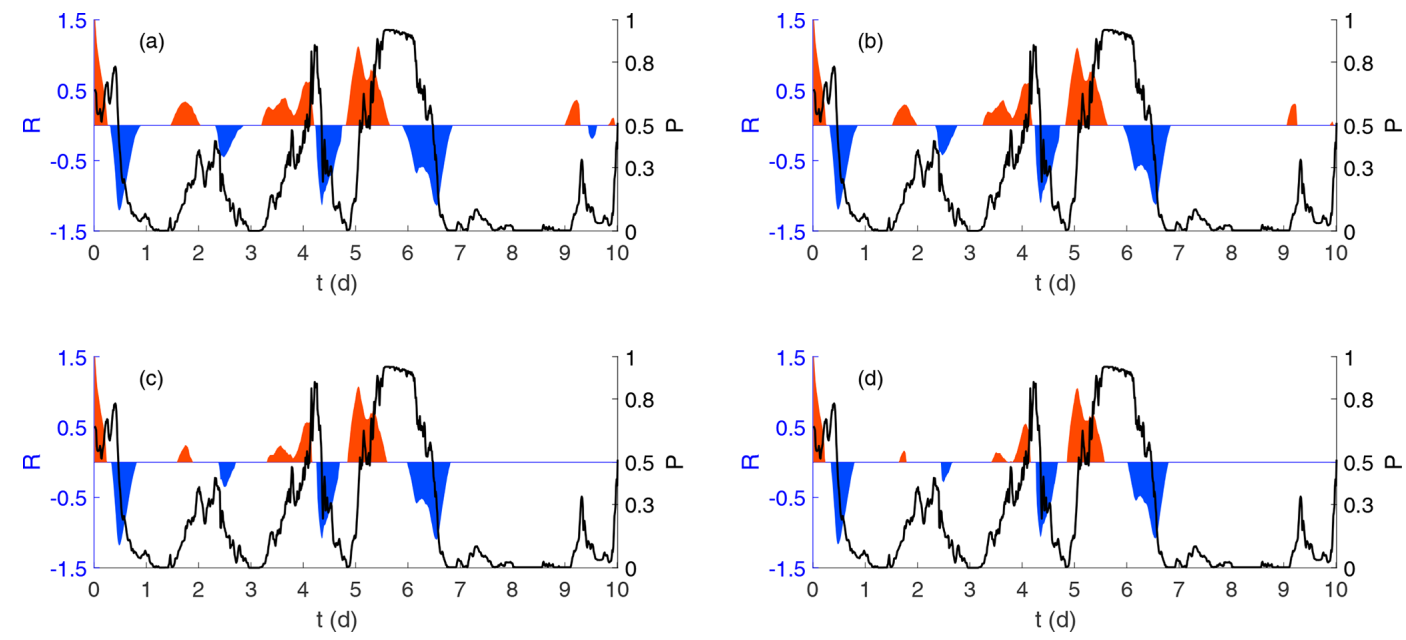

Figure 5. Normalised wind power ramps, $R(t)$, are superimposed over the normalised wind power values $P(t)$ for the $10 \mathrm{~d}$ period discriminated at the $10 \%$ (a), $5 \%$ (b), $2 \%$ (c), and $1 \%$ (d) levels. Ramp-up events are shown in red and ramp-down events in blue.

$R_{\mathrm{u}}^{\prime}(k)=\frac{\sum_{t=1}^{n_{k}} R(t)}{D(k)}$,

$R_{\mathrm{d}}^{\prime}(l)=\frac{\sum_{t=1}^{n_{l}} R(t)}{D(l)}$,

where the $n_{k}$ normalised power ramp-up values $R(t)$ are summed over the duration $D(k)$ of the $k$ th ramp-up event and the $n_{l}$ normalised power ramp-down values $R(t)$ are summed over the duration $D(l)$ of the $l$ th ramp-down event.

\subsection{Overall distributions}

Distribution plots of ramp rates over the entire 2-year period as a function of duration (binned by hour) are shown in Fig. 7. The ramp-up and ramp-down event distributions are broadly similar in nature though there are some features of note:

- There is a strong correlation between the average normalised ramp rate and the duration of the ramp.

- A majority of ramp durations are less than $15 \mathrm{~h}$ with a median value of $8.25 \mathrm{~h}$ for ramp-up events and $8.5 \mathrm{~h}$ for ramp-down events.

- There is a significant spread in ramp rates of duration between 2 and $15 \mathrm{~h}$.

- For ramps of duration greater than around $12 \mathrm{~h}$, ramp rates tend to decrease.

- There are very few ramp events with a duration of longer than a day ( $24 \mathrm{~h})$. 
Table 1. Test periods and length of surrogate series used to detect and quantify ramps.

\begin{tabular}{lllll}
\hline Test period & Start & End & Surrogate period 1 (S1) & $\begin{array}{l}\text { Surrogate } \\
\text { period 2 (S2) }\end{array}$ \\
\hline T1 & 28 Jan 2015 & 7 Feb 2015 & Same as test period & 2015 \\
T2 & 20 Nov 2015 & 30 Nov 2015 & Same as test period & 2015 \\
T3 & 27 Jan 2016 & 5 Feb 2016 & Same as test period & 2016 \\
T4 & 3 Nov 2016 & 13 Nov 2016 & Same as test period & 2016 \\
\hline
\end{tabular}
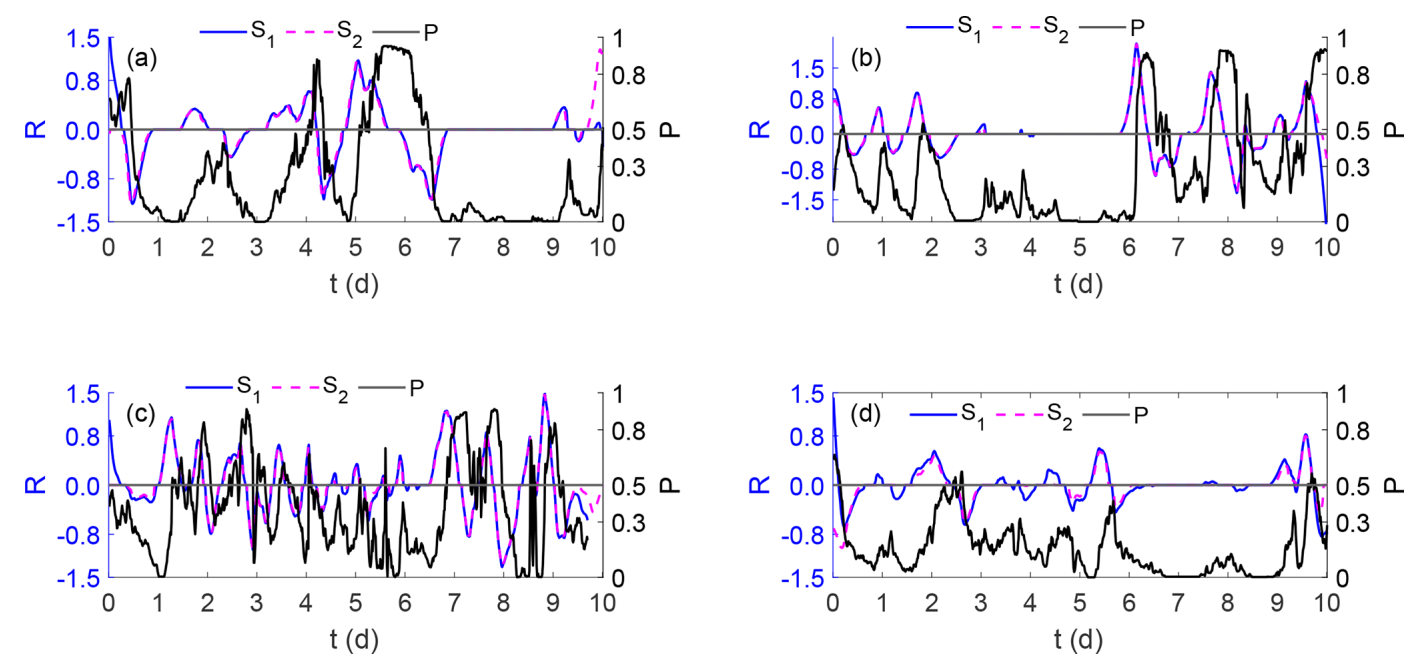

Figure 6. Normalised power ramps: (a) period T1, (b) period T2, (c) period T3, and (d) period T4. Normalised wind power is shown in black. The ramp-ups and ramp-downs derived using surrogate statistics from the $10 \mathrm{~d}$ period (S1) are shown in blue; and those from the 1-year period (S2) are shown in purple.

The features described above are logical when considering the nature of the events driving wind ramps which are generally localised in nature and rarely last longer than a day, such as the passage of a weather front, a sea breeze (Steele et al., 2015), or a low-level jet (Nunalee and Basu, 2014; Kalverla et al., 2019).

\subsection{Diurnal and seasonal dependency}

We also investigate whether the ramp rates show a diurnal or seasonal dependence. To do this, we classify ramp-up and ramp-down rates based on their duration: ramps $\leq 2 \mathrm{~h}$ are classified as short duration; ramps within the range $2-15 \mathrm{~h}$ are classified as medium duration; and long-duration ramps are assumed to be $\geq 15 \mathrm{~h}$. This classification is somewhat arbitrary but is broadly based on the discussion in Sect. 1 . The results are shown in Fig. 8. It can be seen that mediumduration ramps in particular show a strong diurnal cycle with a higher frequency of ramp-up events in the afternoon and ramp-down events in the morning. This is true to a lesser extent for the long-duration ramps. There is no discernible diurnal pattern in the short-duration ramps. By contrast, there is no clear cycle at any scale across the different months of the year. The observed diurnal variation in medium- and long-duration wind power ramps is consistent with the pattern of average diurnal generation observed as seen in Fig. 8e which is strongly influenced by mesoscale effects such as low-level jets, land-sea breezes, and thermally driven entrainment from aloft due to the relatively close proximity of the Belgian offshore wind farms to the coast. Low-level jets are known to be more prevalent during the evening hours at the location of some of the Belgian offshore wind farms (Kalverla et al., 2019) which may contribute to the increase in power generation observed during this period.

These results are based on one dataset for a limited 2-year period. Clearly, further work is necessary to investigate the generality of the observations above. However, this short investigation does illustrate how wavelets can be used to investigate ramps rates, their duration, and their prevalence.

\section{Ramp detection during a period of change in installed power capacity}

As a further illustration of the utility of the ramp detection methodology, we apply it to a different time series of wind power data where the installed capacity increases with time. Such a change can be problematic where ramps are defined 
(a)

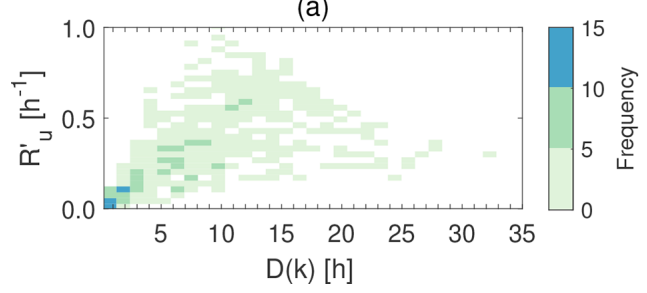

(b)

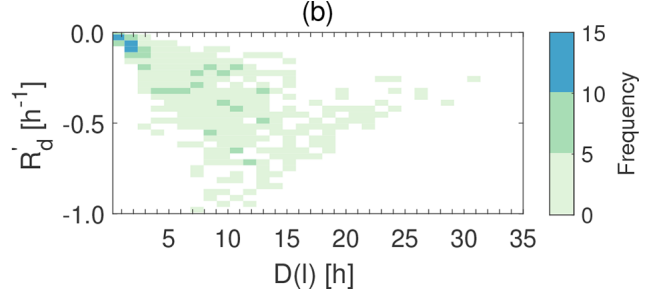

Figure 7. Distributions of normalised ramp rates as a function of duration for (a) ramp-up rates, $R_{\mathrm{u}}^{\prime}(k)$, and (b) ramp-down rates, $R_{\mathrm{d}}^{\prime}(l)$.
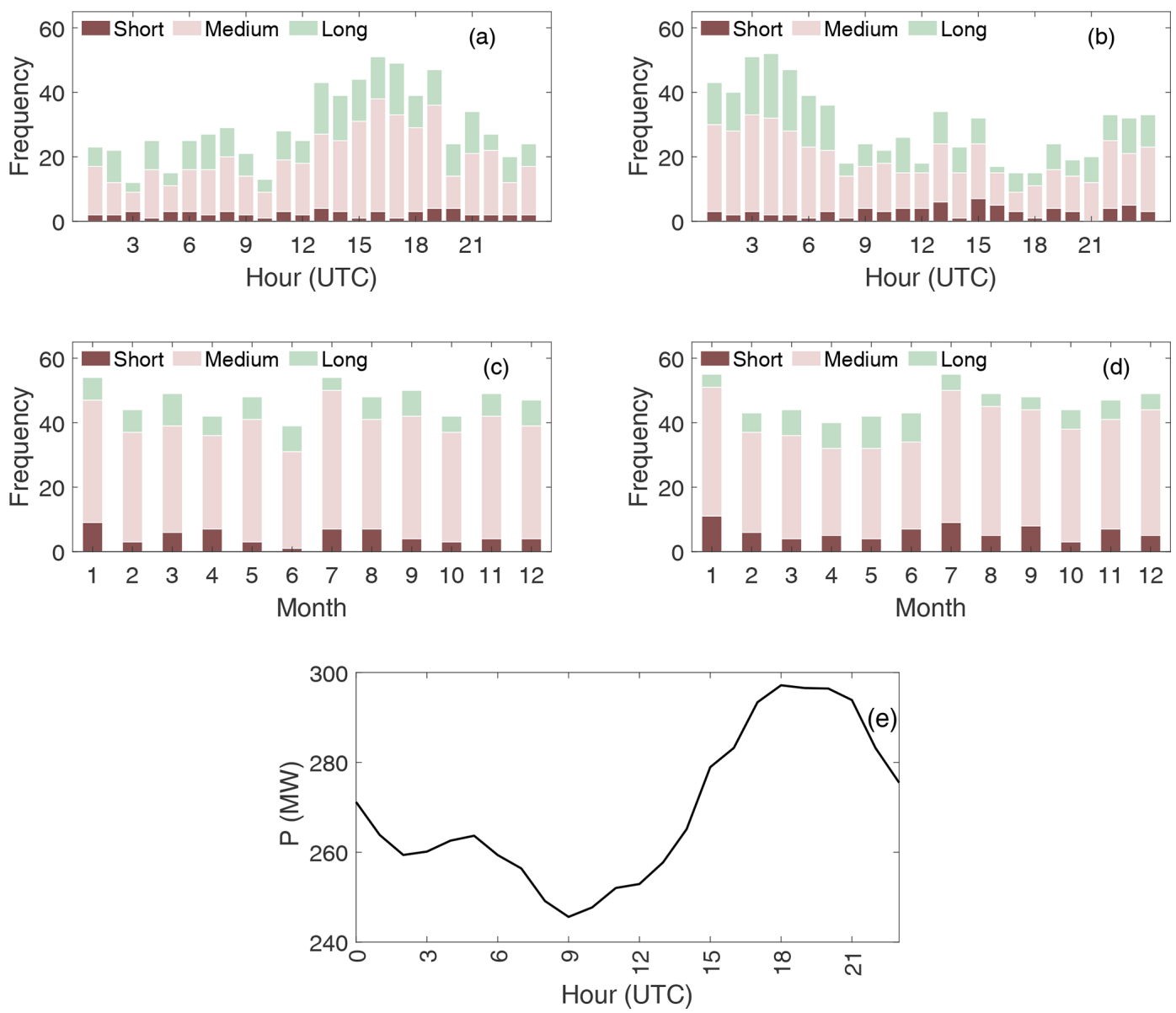

Figure 8. Diurnal and monthly frequency ramps events for short, medium, and long duration: (a) diurnal ramp-up events, (b) diurnal rampdown events, (c) monthly ramp-up events, (d) monthly ramp-down events, (e) diurnal variation in wind power.

using a minimum change in wind power output $\Delta P$ over a time $\Delta t$.

In this section, we use the measured hourly wind power data from the Swedish SE1 price region for the period 20002001. Figure $9 \mathrm{a}$ shows an increasing trend in the production of wind power which reflects an increase in installed capacity over the 2-year period. This trend is also clearly observed in the continuous wavelet coefficient of the power values $(P)$ shown in Fig. 9c. Note that in this case, we have deliberately not normalised the data to show how the method can be applied when the installed capacity is non-stationary. If the wind power data over the period are randomly shuffled, then clearly the trend is no longer visible in either the surrogate series seen in Fig. 9b or its continuous wavelet transform coefficients $W^{*}$ as depicted in Fig. 9d.

We then focus on two periods of data shown by dashed lines in Fig. 9a. In the first period, 1 to 25 January 2000, installed capacity was around $500 \mathrm{MW}$. In the second period, 6 to 31 December 2001, installed capacity had increased to around $1300 \mathrm{MW}$. The difference in magnitude of the $W$ values can clearly be seen in Fig. 10a for the first period and Fig. 10b for the second. Then, using the entire period 2000- 

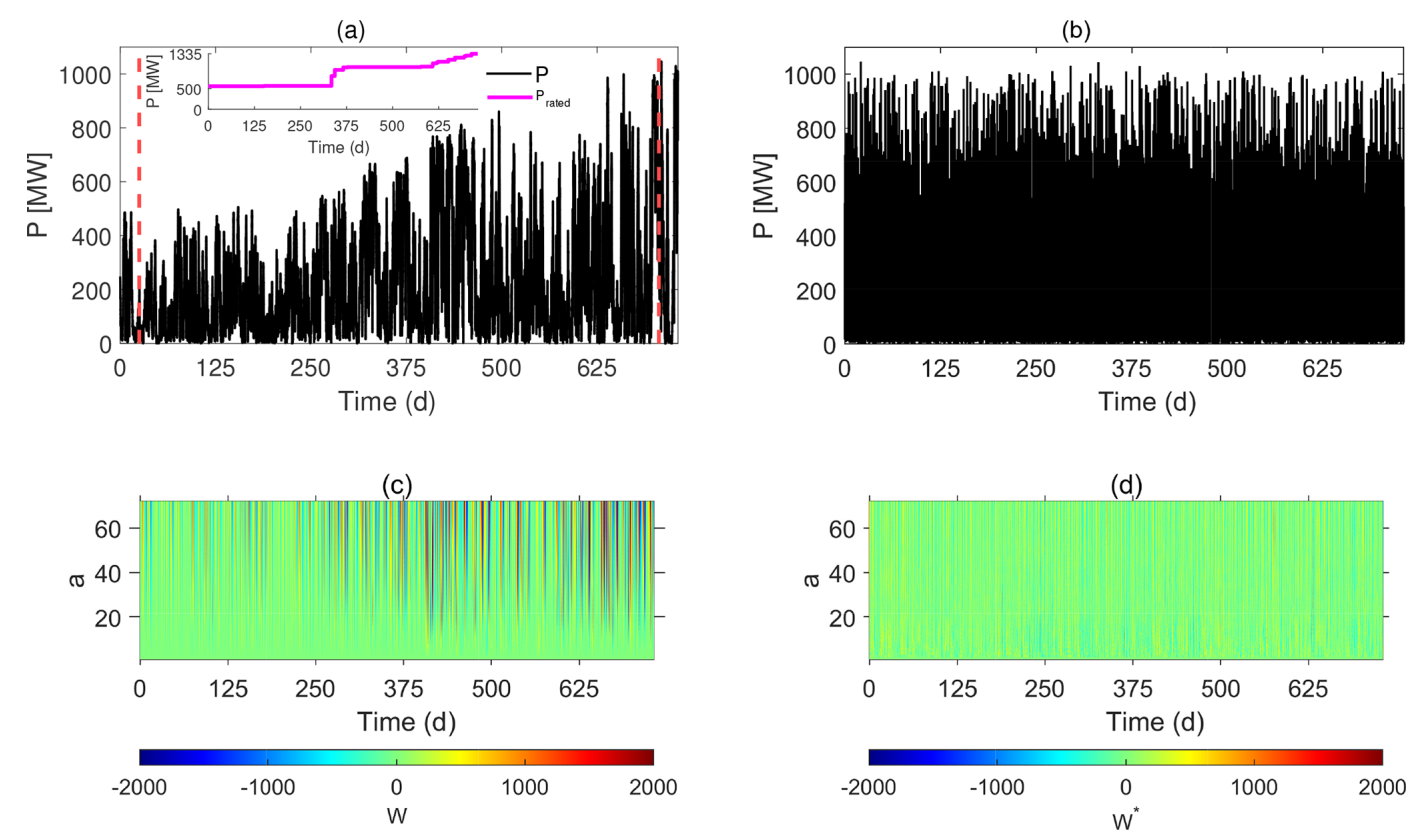

Figure 9. (a) Hourly wind power production $(P)$ and rated power $\left(P_{\text {rated }}\right)$ for the aggregated Swedish energy price region SE1 for $2000-$ 2001. The dashed red lines represent two periods studied in further detail (see Fig. 9). (b) A randomly shuffled hourly values based on the time series in top left; (c) the continuous wavelet transform coefficient of the values in (a); and (d) the continuous wavelet transform coefficient of the randomly shuffled values in (b).

2001 and the method of surrogates to fix the $10 \%$ discrimination level following the same methodology as described above, we calculate the power ramps, $R(t)$, for these two periods. Although the values of $R(t)$ are of a different magnitude for the two periods, it can be seen that there is no discernible difference in the ability to detect ramps in the first period, Fig. 10c, or the second period, Fig. 10d.

\section{A comparison between the wavelet-surrogate and min-max ramp detection methods}

Finally, we compare our wavelet-surrogate (WS) method with an existing ramp detection method known as the minmax method (Bianco et al., 2016). The min-max ramp detection method makes use of a sliding window of a given window length (WL) (in time steps) and considers the change in power during that window defined as the difference between the maximum and minimum power within the window. If this change in power is greater than a defined threshold (TH), then a ramp is deemed to have occurred. Clearly, the sensitivity of this method depends on the chosen values of two parameters, namely WL and TH, in contrast to our WS methodology which depends on a discrimination level. In this study, we compare the two methods in detecting the number of ramp-up and ramp-down events. This comparison is made for both the Belgian and Swedish wind power datasets. Table 2 shows the number of ramp-up and ramp-down events that are detected using the WS and min-max methods for different time periods of the two wind power datasets as a function of different parameter values used by each method. For the WS method, we quantify the sensitivity of ramp detection using the $10 \%$ (WS10), $5 \%$ (WS5), $2 \%$ (WS2), and 1\% (WS1) discrimination levels. In the case of the min-max method, we use a combination of window lengths (WL1 $=8$ and $\mathrm{WL} 2=12$ ) and threshold levels (TH1 $=0.3$ and $\mathrm{TH} 2=0.4$ ). For the $15 \mathrm{~min}$ Belgian offshore wind power data, these WL values correspond to $\mathrm{WL} 1=2 \mathrm{~h}$ and $\mathrm{WL} 2=3 \mathrm{~h}$, whereas for the hourly Swedish onshore wind power data, they correspond to WL1 $=8 \mathrm{~h}$ and $\mathrm{WL} 2=12 \mathrm{~h}$. Note that the actual Swedish wind power data values are used for the WS algorithm, while the data are normalised by time-varying installed power capacity for the min-max method. In general, the number of ramp events detected by the WS method is greater than for the min-max method. The number of events detected by the WS method generally reduces as the discrimination percentage value gets smaller. The exception to this is for the $\mathrm{T} 1$ and $\mathrm{T} 2$ periods, when a slight increase is seen at the $1 \%$ discrimination level when the overall event count is low. This is due to the splitting of single ramp events into two events, an example of which can be seen around day 4 in Fig. 5d. Similarly, the min-max method detects fewer ramp events as TH is increased. By contrast, increasing the WL value does not show such a clear trend in the number of ramp events detected. In addition, the min-max method seems more sensitive to the range of WL and TH values used 

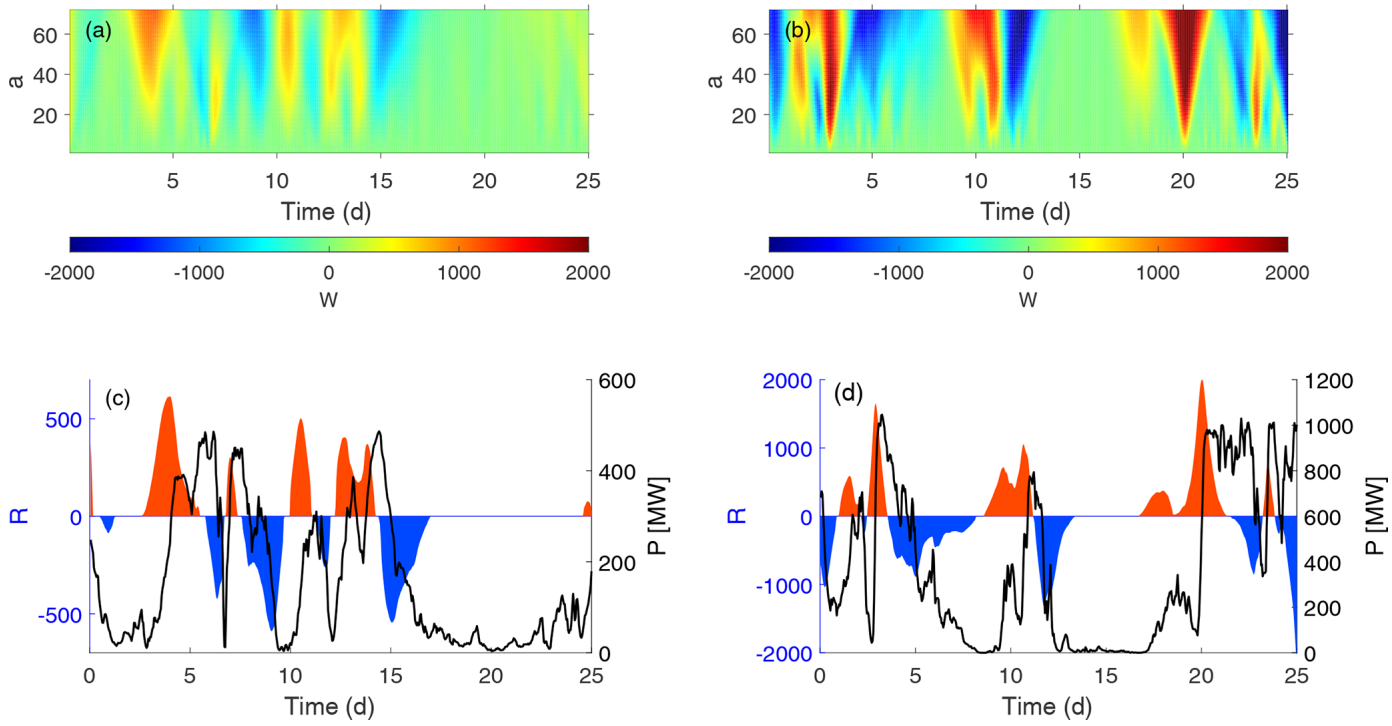

Figure 10. Wavelet coefficients and ramps based on wind power for the aggregated Swedish energy price region SE1 for two periods, 1 to 25 January 2000 and 6 to 31 December 2001; (a) continuous wavelet transform coefficient for the period 1 to 25 January 2000; (b) continuous wavelet transform coefficient for the period 6 to 31 December 2001; (c) power ramps for the period 1 to 25 January 2000 ; and (d) power ramps for the period 6 to 31 December 2001.

Table 2. Comparison of the wavelet-surrogate (WS) and min-max ramp detection methods. The number of ramp-up and ramp-down events are shown in bold and in parenthesis, respectively. Periods T1-T4 are given in Table 1; T5 is the Belgian offshore wind power data for the entire period 2015-2016 and SE1 is the hourly Swedish wind power data for the period 2000-2001.

\begin{tabular}{lrrrr|rrrr}
\hline & \multicolumn{4}{c|}{ WS } & \multicolumn{4}{c}{ Min-max method } \\
\cline { 2 - 8 } Times & WS10 & WS5 & WS2 & WS1 & WL1, TH1 & WL1, TH2 & WL2, TH1 & WL2, TH2 \\
\hline T1 & $\mathbf{6}(6)$ & $\mathbf{6}(5)$ & $\mathbf{4}(4)$ & $\mathbf{6}(4)$ & $\mathbf{4}(2)$ & $\mathbf{1}(2)$ & $\mathbf{4}(3)$ & $\mathbf{4}(3)$ \\
T2 & $\mathbf{1 1}(9)$ & $\mathbf{8}(8)$ & $\mathbf{8}(7)$ & $\mathbf{7}(8)$ & $\mathbf{6}(4)$ & $\mathbf{4}(2)$ & $\mathbf{7}(5)$ & $\mathbf{5}(3)$ \\
T3 & $\mathbf{1 6}(15)$ & $\mathbf{1 4}(13)$ & $\mathbf{1 3}(12)$ & $\mathbf{1 2}(4)$ & $\mathbf{1 5}(13)$ & $\mathbf{1 0}(6)$ & $\mathbf{1 4}(18)$ & $\mathbf{1 1}(10)$ \\
T4 & $\mathbf{1 0}(12)$ & $\mathbf{1 0}(10)$ & $\mathbf{6}(8)$ & $\mathbf{5}(6)$ & $\mathbf{2}(5)$ & $\mathbf{1}(2)$ & $\mathbf{3}(6)$ & $\mathbf{1}(4)$ \\
T5 & $\mathbf{5 6 7}(564)$ & $\mathbf{4 9 8}(487)$ & $\mathbf{4 3 4}(430)$ & $\mathbf{3 9 7}(390)$ & $\mathbf{4 2 6}(386)$ & $\mathbf{2 1 4}(181)$ & $\mathbf{5 2 7}(467)$ & $\mathbf{2 9 4}(260)$ \\
SE1 & $\mathbf{2 3 7}(244)$ & $\mathbf{2 0 9}(220)$ & $\mathbf{1 9 3}(194)$ & $\mathbf{1 7 9}(182)$ & $\mathbf{1 8 5}(179)$ & $\mathbf{8 9}(85)$ & $\mathbf{2 2 4}(224)$ & $\mathbf{1 3 2}(139)$ \\
\hline
\end{tabular}

than the WS method is to the range of discrimination values used.

\section{Conclusions}

The detection of wind power ramps is a challenge in terms of how to characterise their magnitude and duration and how to discriminate a ramp from incoherent stochastic fluctuations in wind power. In this paper, we have presented a relatively simple methodology based on a wavelet transform and the use of surrogates to discriminate and extract ramp events. Using wind power data from the Belgian offshore wind farm cluster, we have illustrated the application of the methodology and have shown that a $10 \mathrm{~d}$ period is sufficient to discriminate coherent ramp events from incoherent fluctuations. We show the utility of the technique in characterising the distribution of ramp rates and their duration and seasonal- diurnal variation for the Belgian offshore cluster. In addition, we have shown how the methodology can be used to detect wind power ramps when installed capacity increases with time using Swedish onshore wind power data as an example. Lastly, we compare our ramp detection algorithm with the min-max method, contrasting their sensitivities to parameter choice. Further work is required to apply the methodology to a broader range of sites and for longer periods to investigate the prevalence of different ramp rates and their duration. It might be expected that depending on the climatology of the site, this could differ; on the other hand, consistent trends may be apparent which could help operators in accommodating fluctuations within an integrated power system. 
Data availability. The datasets are freely available at https://www. elia.be/en/grid-data/power-generation/wind-power-generation (Elia, 2020) and https://eem20.eu/forecasting-competition/ (EEM20, 2020).

Author contributions. This research was carried out by BRC under the supervision of SJW and SB.

Competing interests. The authors declare that they have no conflict of interest.

Acknowledgements. The authors are grateful to Elia for supplying the Belgian offshore wind farm production data and the KTH Royal Institute of Technology-Greenlytics for supplying the Swedish onshore wind farm production data used in this research.

Review statement. This paper was edited by Julie Lundquist and reviewed by two anonymous referees.

\section{References}

Bianco, L., Djalalova, I. V., Wilczak, J. M., Cline, J., Calvert, S., Konopleva-Akish, E., Finley, C., and Freedman, J.: A wind energy ramp tool and metric for measuring the skill of numerical weather prediction models, Weather Forecast., 31, 1137-1156, 2016.

Borgnat, P. and Flandrin, P.: Stationarization via surrogates, J. Stat. Mech.: Theory and Experiment, 2009, P01001, https://doi.org/10.1088/1742-5468/2009/01/p01001, 2009.

Borgnat, P., Flandrin, P., Honeine, P., Richard, C., and Xiao, J.: Testing stationarity with surrogates: A time-frequency approach, IEEE Transactions on Signal Processing, 58, 3459-3470, 2010.

Bossavy, A., Girard, R., and Kariniotakis, G.: Forecasting Uncertainty Related to Ramps of Wind Power Production, European Wind Energy Conference and Exhibition 2010, EWEC 2010, April 2010, Warsaw, Poland, 9 pp., ISBN 9781617823107.Hal00765885 f, 2010.

Coughlin, K., Murthi, A., and Eto, J.: Multi-scale analysis of wind power and load time series data, Renew. Energ., 68, 494-504, 2014.

Cutler, N., Kay, M., Jacka, K., and Nielsen, T. S.: Detecting, categorizing and forecasting large ramps in wind farm power output using meteorological observations and WPPT, Wind Energy: An International Journal for Progress and Applications in Wind Power Conversion Technology, 10, 453-470, 2007.

Davy, M. and Godsill, S.: Detection of abrupt spectral changes using support vector machines an application to audio signal segmentation, 2002 IEEE International Conference on Acoustics, Speech, and Signal Processing, Orlando, FL, 2002, II-1313-II1316, https://doi.org/10.1109/ICASSP.2002.5744044, 2002.

DeMarco, A. and Basu, S.: On the tails of the wind ramp distributions, Wind Energ., 21, 892-905, 2018.
Drew, D. R., Barlow, J. F., and Coker, P. J.: Identifying and characterising large ramps in power output of offshore wind farms, Renew. Energ., 127, 195-203, 2018.

Dunyak, J., Gilliam, X., Peterson, R., and Smith, D.: Coherent gust detection by wavelet transform, J. Wind Eng. Ind. Aerod., 77, 467-478, 1998.

EEM20: EEM20 Forecasting Competition, available at: https: //eem20.eu/forecasting-competition/ (last access: 17 August 2020), 2020.

Elia: Wind power generation, available at: https://www.elia.be/en/ grid-data/power-generation/wind-power-generation (last access: 17 August 2020), 2020.

Ferreira, C., Gama, J., Matias, L., Botterud, A., and Wang, J.: A Survey on Wind Power RAMP Forecasting, Tech. rep., Argonne National Laboratory (ANL), USA, 2011.

Gallego, C., Cuerva, A., and Costa, A.: Detecting and characterising ramp events in wind power time series, Journal of Physics: Conference Series, 555, 012040, https://doi.org/10.1088/17426596/555/1/012040, 2014.

Gallego-Castillo, C., Cuerva-Tejero, A., and Lopez-Garcia, O.: A review on the recent history of wind power ramp forecasting, Renewable and Sustainable Energy Reviews, 52, 1148-1157, 2015.

Gilliam, X., Dunyak, J., Doggett, A., and Smith, D.: Coherent structure detection using wavelet analysis in long time-series, J. Wind Eng. Ind. Aerod., 88, 183-195, 2000.

Guarin, D., Orozco, A., and Delgado, E.: A new surrogate data method for nonstationary time series, arXiv [preprint], arXiv:1008.1804, 2010.

Hannesdóttir, Á. and Kelly, M.: Detection and characterization of extreme wind speed ramps, Wind Energ. Sci., 4, 385-396, https://doi.org/10.5194/wes-4-385-2019, 2019.

Ji, F., Cai, X., and Zhang, J.: Wind power prediction interval estimation method using wavelet-transform neuro-fuzzy network, Journal of Intelligent \& Fuzzy Systems, 29, 2439-2445, 2015.

Kalverla, P. C., Duncan Jr., J. B., Steeneveld, G.-J., and Holtslag, A. A. M.: Low-level jets over the North Sea based on ERA5 and observations: together they do better, Wind Energ. Sci., 4, 193209, https://doi.org/10.5194/wes-4-193-2019, 2019.

Kiviluoma, J., Holttinen, H., Scharff, R., Weir, D. E., Cutululis, N., Litong-Palima, M., and Milligan, M.: Index for wind power variability, in: The 13th Wind Integration Workshop, edited by: Betancourt, U. and Ackermann, T., 11-14 November 2014, Berlin, 2014.

Kiviluoma, J., Holttinen, H., Weir, D., Scharff, R., Söder, L., Menemenlis, N., Cutululis, N. A., Danti Lopez, I., Lannoye, E., Estanqueiro, A., Gomez-Lazaro, E., Zhang, Q., Bai, J., Wan, Y.-H., and Milligan, M.: Variability in large-scale wind power generation, Wind Energ., 19, 1649-1665, 2016.

Laurent, H. and Doncarli, C.: Stationarity index for abrupt changes detection in the time-frequency plane, IEEE Signal Processing Letters, 5, 43-45, 1998.

Mallat, S.: A Wavelet Tour of Signal Processing. A Wavelet Tour of Signal Processing, Academic Press, ISBN 13 978-0-12-374370$1,2009$.

Marquis, M., Wilczak, J., Ahlstrom, M., Sharp, J., Stern, A., Smith, J. C., and Calvert, S.: Forecasting the wind to reach significant penetration levels of wind energy, B. Am. Meteorol. Soc., 92, 1159-1171, 2011. 
Nunalee, C. and Basu, S.: Mesoscale Modeling of Low-Level Jets over the North Sea, in: Wind Energy - Impact of Turbulence, edited by: Hölling, M., Peinke, J., and Ivanell, S., pp. 197-202, Springer Berlin Heidelberg, Berlin, Heidelberg, 2014.

SCB: Energy prices and switching of suppliers, 3rd quarter 2017, Tech. Rep. Technical Report EN 24 SM 1704, Swedish Energy Agency, 2017.

Sevlian, R. and Rajagopal, R.: Detection and statistics of wind power ramps, IEEE Transactions on Power Systems, 28, 3610 $3620,2013$.
Steele, C., Dorling, S., von Glasow, R., and Bacon, J.: Modelling sea-breeze climatologies and interactions on coasts in the southern North Sea: implications for offshore wind energy, Q. J. Roy. Meteorol. Soc., 141, 1821-1835, 2015.

Theiler, J., Eubank, S., Longtin, A., Galdrikian, B., and Farmer, J. D.: Testing for nonlinearity in time series: the method of surrogate data, Physica D: Nonlinear Phenomena, 58, 77-94, 1992.

Zhang, J., Cui, M., Hodge, B.-M., Florita, A., and Freedman, J.: Ramp forecasting performance from improved short-term wind power forecasting over multiple spatial and temporal scales, Energy, 122, 528-541, 2017. 